\title{
Opiniones de los inspectores médicos acerca de la distorsión clínica en la valoración de la incapacidad laboral en España.
}

\author{
Antonio Domínguez Muñoz ${ }^{1,2^{*}}$, Ana Ma Gómez Álvarez ${ }^{1}$, José Luis de la Fuente Madero ${ }^{1}$, Ma José López Pérez ${ }^{2,3}$ \\ Pablo García Ruiz ${ }^{4}$, Rafael López Pérez ${ }^{5}$
}

Resumen: Introducción: El contexto médico-legal en el que se desarrolla la medicina evaluadora, permitiendo el acceso a prestaciones económicas, obliga a considerar la validez en las consultas de valoración médica de incapacidad para tratar de evitar el fraude por simulación. Por otra parte, en el ámbito médico asistencial son habituales los casos biomédicamente inexplicables que se acompañan de elevados niveles subjetivos de sufrimiento, para los que se ha propuesto el término MUPS (medically unexplained physical symptoms). Objetivos: Profundizar en el fenómeno de la distorsión clínica en la valoración médica de incapacidad, basado en los dos primeros criterios de Simulación del DSM-IV-TR, referido al marco teórico del Análisis de Conducta en Medicina. Métodos: Encuesta en línea dirigida al colectivo de los inspectores médicos evaluadores del Instituto Nacional de la Seguridad Social (INSS) de España para conocer sus opiniones acerca de estos casos, presentes en su práctica diaria, su manejo de los mismos y su grado de concordancia con respecto a la bibliografía de referencia. Resultados: Incluyen a una proporción representativa de dichos inspectores médicos (15\%), que aparecen como un colectivo variado, por su distinta formación profesional, experiencia previa y práctica diaria en las diferentes Unidades Médicas. Conclusiones: Consideran la distorsión clínica y sus cuadros anexos como situaciones frecuentes y relevantes en sus consultas, principalmente el fraude por simulación. Pese a ello, los inspectores médicos no parecen contar actualmente con referencias específicas suficientes ni con un discurso científico común estructurado para estos casos, que son abordados según criterios individuales más o menos ajustados al estado actual del conocimiento.

Palabras clave: distorsión clínica; fraude por simulación; baja laboral; somatización; valoración médica de la incapacidad; análisis de conducta.

Abstract: Introduction: The forensic context in which disability assessment medicine is practiced in Spain, allowing access to workers' compensation benefits, requires consideration of the validity of disability assessment examinations. On the other hand, in clinical medicine biomedically unexplained cases -for which the term medically unexplained physical symptoms (MUPS) was proposed- are common, often accompanied by high levels of subjective suffering. Aim: To deepen in the phenomenon of clinical distortion in disability assessment examinations, based on the first two criteria of Malingering in DSM-IV-TR, referring to the theoretical framework of the Behavior Analysis in Medicine. Methods: Online survey addressed to the group of medical examiners of the National Institute of Social Security (INSS) of Spain to know their opinions on these cases, present in their everyday practice, their management and the extent of agreement to the literature of reference. Results: Collected a representative proportion of medical examiners (15\%), they appear as a miscellaneous group due to their different professional training, previous experience and everyday practice in their different Medical Units. Conclusions: medical examiners consider the clinical distortion and related cases as frequent and relevant situations in their practice, mainly malingering. Nonetheless, medical examiners do not seem to have sufficient specific references or a structured common scientific discourse for these cases, which are approached according to individual criteria more or less adjusted to the current state of knowledge.

Keywords: clinical distortion; malingering; sickness leave; somatization; disability assessment medicine; behavior analysis in medicine.

Fecha de envío: 21 de enero de 2017 - Fecha de aceptación: 28 de abril de 2017

(1) Unidad Médica del Equipo de Valoración de Incapacidades (UMEVI) de la Dirección Provincial del Instituto Nacional de la Seguridad Social (INSS) de Málaga (España).

(2) Departamento de Análisis de Conducta en Medicina de la Fundación Universitaria Behavior and Law.

(3) Centro Médico Valdebernardo de Madrid (España).

(4) Unidad Médica del Equipo de Valoración de Incapacidades (UMEVI) de la Dirección Provincial del Instituto Nacional de la Seguridad Social (INSS) de Granada (España).

(5) Fundación Universitaria Behavior and Law.

*Autor de correspondencia: adominguez@behaviorandlaw.com 


\section{Introducción}

La medicina pericial o evaluadora se encargaría de estudiar, verificar, cuantificar y describir procesos patológicos, congénitos o adquiridos, tanto físicos como psíquicos, con el fin de determinar la posible repercusión orgánica, funcional, laboral, económica y legal. Uno de sus campos es la valoración médica de la incapacidad laboral en relación con las prestaciones contributivas de Seguridad Social (Arancón, 2004), situación que en España, dentro del ámbito de la Administración General del Estado, se lleva a cabo por las llamadas UMEVI-unidad médica del equipo de valoración de incapacidades-, adscritas a las direcciones provinciales del Instituto Nacional de la Seguridad Social (INSS), donde actúan los inspectores médicos evaluadores como peritos titulares (Álvarez et al., 2003) .

La existencia de un contexto médico-legal como el presente en la Seguridad Social, que proporciona acceso a prestaciones económicas, implica considerar la validez de las consultas de valoración de incapacidad, dentro de su complejidad administrativa (Burgos et al., 2008) analizando la posible distorsión orientada a obtener o prolongar prestaciones, sea de manera fraudulenta o no.

A los efectos de este trabajo y como modo de abordar dicha validez, se ha considerado la presencia de distorsión clínica cuando están presentes los dos primeros criterios de fraude por simulación (malingering) del DSM-IV-TR (American Psychiatric Association. Task Force on DSM-IV., 2000) ; es decir, la existencia de una clara desproporción entre los elementos objetivos y subjetivos del caso, en un contexto médico-legal. También, más específicamente, si se observa la presencia de un estilo de respuesta magnificador, reductor o combinado de los descritos por Tearnan (2003). Con "estilo de respuesta" dicho autor hace referencia a los patrones de comportamiento presentados por los pacientes cuando informan de sus síntomas, físicos o psíquicos. Tearnan se basa en Rogers (2008) y considera básicamente cinco estilos de respuesta; Sincero (Honest), Magnificador (Maximization), Reductor (Minimization), Combinado (Mixed) e Irrelevante (Irrelevant).

La distorsión clínica así definida puede ser voluntaria o no; aquella que lo sea y esté orientada a obtener un determinado objetivo puede considerarse engaño (deception) y solo el engaño que busca un beneficio externo correspondería a fraude por simulación ( $m a-$ lingering). Si el engaño responde a una necesidad intrapsíquica de asumir el rol de enfermo, correspondería a un trastorno facticio, según los criterios del DSM-IV-TR. Por otro lado, la distorsión puede ser involuntaria como ocurriría con los trastornos somatomorfos, que incluyen entre otros los conversivos y la somatización, presentando síntomas físicos o psíquicos que están fuera del control consciente del paciente y corresponden a manifestaciones físicas de origen psicoemocional. El resumen de este diagnóstico diferencial básico de la distorsión clínica se recoge en la Figura 1, y en trabajos recientes ya se ha tratado la utilidad de su abordaje en consulta usando un abordaje interdisciplinar, basado en la aplicación del análisis de conducta (Domínguez, 2016), a la medicina.

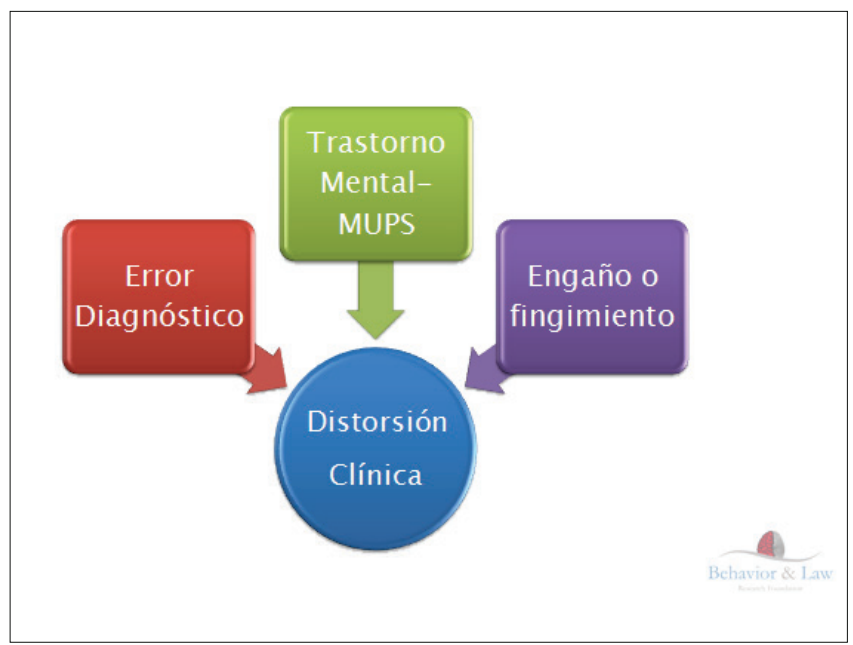

Figura 1: Esquema propuesto para el diagnóstico diferencial básico de la Distorsión Clínica (Domínguez, 2016). Los MUPS -medically unexplained physical symptoms- serían el equivalente a la distorsión en un contexto médico asistencial y suelen tener como base la somatización (Richardson \& Engel, 2004).

\section{Objetivos}

El objetivo general de este trabajo es profundizar en el conocimiento del fenómeno de la distorsión clínica y su manejo en la valoración médica de la incapacidad laboral en las Unidades Médicas de la Seguridad Social de España, mediante la encuesta al colectivo de los inspectores médicos evaluadores del INSS.

Los objetivos específicos serían:

1. Recoger las opiniones de los médicos que trabajan en el ámbito de la valoración de incapacidad en el INSS con respecto a diferentes aspectos de la distorsión clínica, que -de forma simplificada- incluiría aquellos casos en los que se observan acusadas discrepancias entre los elementos clínicos objetivos y la afectación o las limitaciones funcionales mostradas. Los principales diagnósticos asociados a la distorsión clínica, como hemos explicado, serían los trastornos somatomorfos, los de conversión, los facticios y el fraude por simulación.

2. Analizar las opiniones obtenidas, su grado de concordancia y ajuste al estado actual del conocimiento y a las principales referencias doctrinales en Medicina Evaluadora. 


\section{Métodos}

Se realizó una encuesta en línea, anónima y voluntaria, usando un formulario de Google (http://goo.gl/oLWmR) que incluye 35 apartados. Tras los siete primeros, Datos Biográficos del encuestado, los veinte siguientes corresponden a Proposiciones -sobre aspectos diversos- que deben ser valoradas mediante una escala numérica (0-10) de desacuerdo-acuerdo. Los últimos ocho apartados incluyen seis preguntas concretas acerca de la práctica diaria, a responder en campo de texto y dos preguntas finales con opciones de respuesta múltiple. Se solicitó mediante correo electrónico a los médicos evaluadores que, de manera voluntaria, respondiesen dicho cuestionario en línea y se realizó posteriormente un análisis estadístico muy básico tras considerar completo el periodo de recogida de datos, a finales de 2012.

Se revisaron los textos de referencia en Medicina Evaluadora disponibles con respecto al análisis de validez y los principales diagnósticos asociados a la distorsión clínica; el Manual de actuación para médicos del INSS (2003; 2011), la Guía de Valoración de Incapacidad Laboral para Médicos de Atención Primaria (Álvarez et al., 2009), así como el programa formativo de las dos últimas ediciones del Curso de Actualización para médicos evaluadores del INSS, correspondientes a 2011, cuando se celebraba una edición anual, y del bienio 2012-2013.

\section{Resultados y discusión}

Datos biográficos: Se obtuvieron 89 respuestas de inspectores médicos evaluadores del INSS en activo, lo que representa un 15\% del colectivo, cuyo número aproximado se acerca a las seiscientas personas. Un $65 \%$ eran mujeres, con una edad media de $48,2 \pm 6,6$ años y unos 24 años de ejercicio profesional. Los hombres tenían una edad algo superior, $52,7 \pm 5,7$ años y unos 28 años de experiencia.

Respecto a su formación previa, la mayoría eran especialistas en Medicina del Trabajo, -28 personas- o en Medicina Familiar y Comunitaria -25 personas-. Solo había 22 inspectores médicos sin especialidad, lo que supone un $25 \%$ del grupo mientras que otro 15\% tenía alguna otra especialidad, estando representadas desde la M. Interna (3) a los Análisis Clínicos (2), así como Alergia o Traumatología.

Como experiencia asistencial previa a la Inspección, un 33\% de los inspectores médicos contaba con práctica asistencial hospitalaria y un $27 \%$ había prestado servicios -asistenciales o periciales- en una Mutua, entidades sin ánimo de lucro que gestionan cotizaciones de la Seguridad Social tanto en accidentes de trabajo y enfermedades profesionales (Vicente et al., 2012) como en contingencias comunes. Había más de cuarenta inspectores con formación
Tabla 1: Con las 20 proposiciones planteadas para su valoración en escala de 0 a 10.

\begin{tabular}{|c|c|c|}
\hline No & Proposición & Subgrupo \\
\hline P1 & $\begin{array}{l}\text { En mi ejercicio profesional actual me encuentro } \\
\text { habitualmente con distorsión clínica, es decir, } \\
\text { pacientes que alteran o exageran su cuadro } \\
\text { clínico real y/o las limitaciones que producen. }\end{array}$ & $\begin{array}{l}\text { Práctica } \\
\text { Diaria }\end{array}$ \\
\hline P2 & $\begin{array}{l}\text { Dicha distorsión es fácil de notar pero difícil de } \\
\text { explicar. }\end{array}$ & P. Diaria \\
\hline P3 & $\begin{array}{l}\text { Normalmente, corresponde a una exageración } \\
\text { intencionada de los síntomas o las limitaciones } \\
\text { con un claro objetivo material. }\end{array}$ & P. Diaria \\
\hline P4 & $\begin{array}{l}\text { Si la detecto, incluyo en la historia clínica } \\
\text { referencias a la posible distorsión o los potenciales } \\
\text { beneficios secundarios. }\end{array}$ & P. Diaria \\
\hline P5 & $\begin{array}{l}\text { En mi práctica diaria suelo centrarme en } \\
\text { los aspectos asistenciales (diagnósticos y } \\
\text { terapéuticos) y no en valorar la posible distorsión. }\end{array}$ & P. Diaria \\
\hline P6 & $\begin{array}{l}\text { Creo que si un paciente me miente en consulta } \\
\text { para conseguir algo yo ni puedo ni debo hacer de } \\
\text { detective. }\end{array}$ & $\begin{array}{l}\text { Aspectos } \\
\text { Básicos }\end{array}$ \\
\hline P7 & $\begin{array}{l}\text { Dedicarme a detectar una eventual distorsión } \\
\text { o simulación sería ir en contra del bien de mi } \\
\text { paciente y, por lo tanto, del código deontológico } \\
\text { médico. }\end{array}$ & A. Básicos \\
\hline P8 & $\begin{array}{l}\text { De hecho, la distorsión en mi consulta es rara y } \\
\text { la simulación (engaño deliberado para conseguir } \\
\text { beneficios económicos o materiales) aún más. }\end{array}$ & A.. Básicos \\
\hline P9 & $\begin{array}{l}\text { la simulación, el paciente está sano y quiere } \\
\text { sar por enfermo. Si está enfermo, ya no es un } \\
\text { nulador. }\end{array}$ & A. Básicos \\
\hline P10 & $\begin{array}{l}\text { compruebo que el paciente me miente en la } \\
\text { nnsulta, sabré que es un simulador. }\end{array}$ & Controversias \\
\hline P11 & $\begin{array}{l}\text { Si detecto que el paciente finge o exagera en } \\
\text { consulta, sabré que es un simulador. }\end{array}$ & Diagnóstico \\
\hline P12 & $\begin{array}{l}\text { No existen maniobras en la exploración física que } \\
\text { nos permitan comprobar que un paciente está } \\
\text { fingiendo. }\end{array}$ & Diagnóstico \\
\hline P13 & $\begin{array}{l}\text { Los signos de Waddell nos permiten detectar } \\
\text { distorsión, pero no simulación, en la exploración } \\
\text { física. }\end{array}$ & Diagnóstico \\
\hline P14 & $\begin{array}{l}\text { La simulación se observa en una proporción de } \\
\text { casos similar con independencia del contexto } \\
\text { médico: asistencial, forense, valoración de } \\
\text { incapacidad }\end{array}$ & Diagnóstico \\
\hline P15 & $\begin{array}{l}\text { No es posible saber de manera científicamente } \\
\text { válida y fiable si la distorsión es voluntaria o no. }\end{array}$ & Controversias \\
\hline P16 & $\begin{array}{l}\text { La detección de un paciente simulador es } \\
\text { relativamente sencilla para alguien con } \\
\text { experiencia clínica asistencial. }\end{array}$ & Diagnóstico \\
\hline P17 & $\begin{array}{l}\text { La simulación es un acto fraudulento llevado a } \\
\text { cabo por una persona antisocial. }\end{array}$ & Controversias \\
\hline P18 & $\begin{array}{l}\text { La simulación es un signo de enfermedad, llevado } \\
\text { a cabo por un enfermo mental. }\end{array}$ & Controversias \\
\hline P19 & $\begin{array}{l}\text { Solo cabe pensar en simulación si hay elevados } \\
\text { incentivos económicos. }\end{array}$ & A. Básicos \\
\hline P20 & $\begin{array}{l}\text { La simulación se produce cuando una persona } \\
\text { está en una situación difícil y no encuentra una } \\
\text { opción mejor para afrontarla. Todos podemos } \\
\text { simular si se dan las condiciones adecuadas. }\end{array}$ & Controversias \\
\hline
\end{tabular}


de postgrado -títulos de experto o maestría- en valoración del daño corporal y pericia médica, si bien solo tres de ellos habían ejercido como peritos privados en procesos judiciales antes de acceder a su actual puesto de inspector médico.

Respuesta a las preguntas: En cuanto a su actividad clínica, los inspectores médicos reciben en consulta una media de 48 trabajadores semanales, con un amplio rango, probablemente en función del tipo de expedientes y su complejidad. Usando la media de sus respuestas, la distorsión clínica estaría presente en el 37\% de los casos, la somatización en el $23 \%$ (19) y la simulación en el $27 \%$ (22), siendo menor el todo que la suma de las partes. Si usamos las modas, los valores son -respectivamente-, el $40 \%$, el $10 \%$ y el $10 \%$, lo que resulta más congruente aunque creemos que mucho menos ajustado a la realidad, dado el bien conocido contexto de la valoración de incapacidad (Aronoff, 1991).

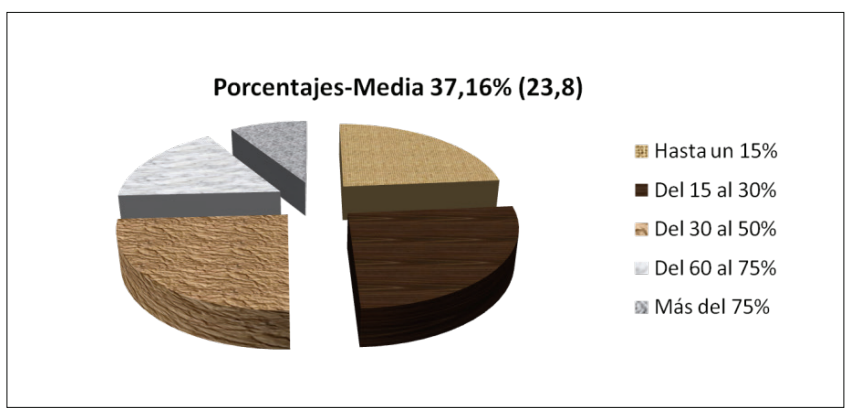

Figura 2: Distribución de respuestas sobre la proporción de casos de distorsión clínica.

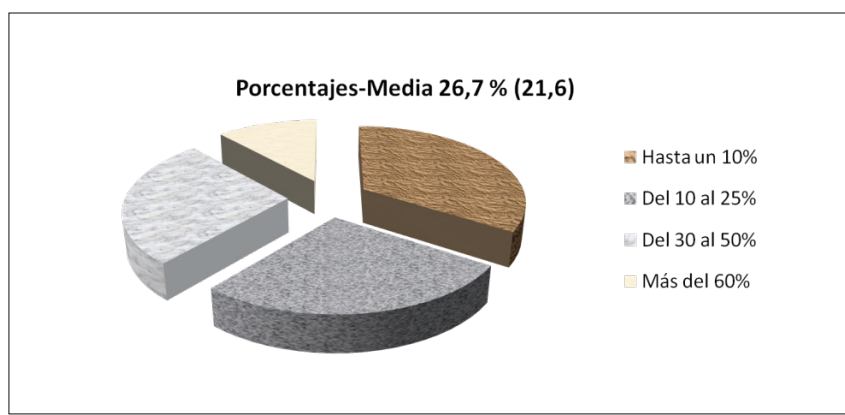

Figura 3: Distribución de respuestas sobre la proporción de casos de simulación.

Dicha estimación sobre distorsión clínica -entendida básicamente como una impresión subjetiva de desproporción entre los elementos objetivos y la afectación subjetiva mostrada en consulta de valoración de incapacidad- sería muy inferior a la que publicamos en un estudio previo (Domínguez et al., 2013). Así, de un grupo de cien trabajadores que recibieron el alta laboral por Inspección, solo siete mostraban un estilo de respuesta sincero. El resto se distribuían en distorsión ligera (25 casos), moderada (41) y severa (27). La duración media de la baja aumentaba a medida que lo hacía la distorsión.
Proposiciones (P): Las 20 proposiciones -ver Tabla 1.- se distribuyen en cuatro categorías; Aspectos Básicos, Controversias, Diagnóstico y Práctica Diaria y los principales resultados están plasmados gráficamente en diez gráficos, numerados como la Proposición que representan.

En general, observamos una tendencia al acuerdo acerca de los aspectos mejor conocidos de la distorsión clínica y sus subtipos, lo que concentra las respuestas de los inspectores médicos en uno de los lados de la escala propuesta de acuerdo/desacuerdo como observamos en las gráficas P1, P3 y P4, correspondientes a la categoría de Aspectos Básicos. Por otro lado, existen proposiciones -P10 y P15 de Controversias o P13 y P16 de Diagnóstico- cuyas respuestas más seleccionadas son las situadas en el centro de la escala -valores 5 y 6- equivalentes a "No sabe/No contesta", mientras que en otras aparece una distribución muy amplia de las respuestas, lo que correspondería con la existencia de discrepancias significativas entre los inspectores médicos sobre aspectos relevantes de los temas tratados, por lo que haremos algunas consideraciones al respecto.

En primer lugar, en el grupo de las respuestas dicotómicas, lo que muestra el gráfico de la Proposición 3 -acerca de incluir en la historia los elementos relativos a la distorsión y sus motivaciones- nos parece un ejemplo de respuesta basada en la deseabilidad social, entendida como la tendencia a presentarse ante los demás de una forma adecuada desde el punto de vista social, es decir, de la forma más valorada socialmente (Briñol et al., 2001). Dado que es habitual compartir casos durante los procesos de incapacidad temporal (IT) en los que suele haber varias consultas, hemos comprobado en los años inmediatamente previos a esta encuesta que -en general- hay muy escasas referencias a las cuestiones relativas a la distorsión clínica, el análisis de validez en la valoración de incapacidad y anexas en la historia clínica principal de los aplicativos informáticos de las Unidades Médicas. Habría algunas excepciones, pero estarían recogidas en el espacio que la Ley de Autonomía del Paciente, que reconoce su derecho a acceder a la historia clínica (Ley 41/2002) reserva para las anotaciones subjetivas del médico, que sólo otros médicos podrían consultar. Hay que recordar que estas anotaciones, aunque visibles para los inspectores médicos mientras el caso está activo, no serían parte del expediente que se remitiría al trabajador o al juzgado en caso de litigio y, por ello, estarían ausentes de cualquier consideración externa a la propia Unidad Médica. Una de las explicaciones, además de la obvia incomodidad del asunto, es la dificultad para encontrar la terminología adecuada para describir lo que observamos en las consultas de manera que sea apropiado y compartido por el resto de los médicos peritos, sean o no inspectores (Domínguez, 2014). El modelo de análisis de validez que proponemos, que parte de la distorsión clínica, incorpora una terminología accesible, sencilla y basada en elementos presentes en la bibliografía de referencia. 


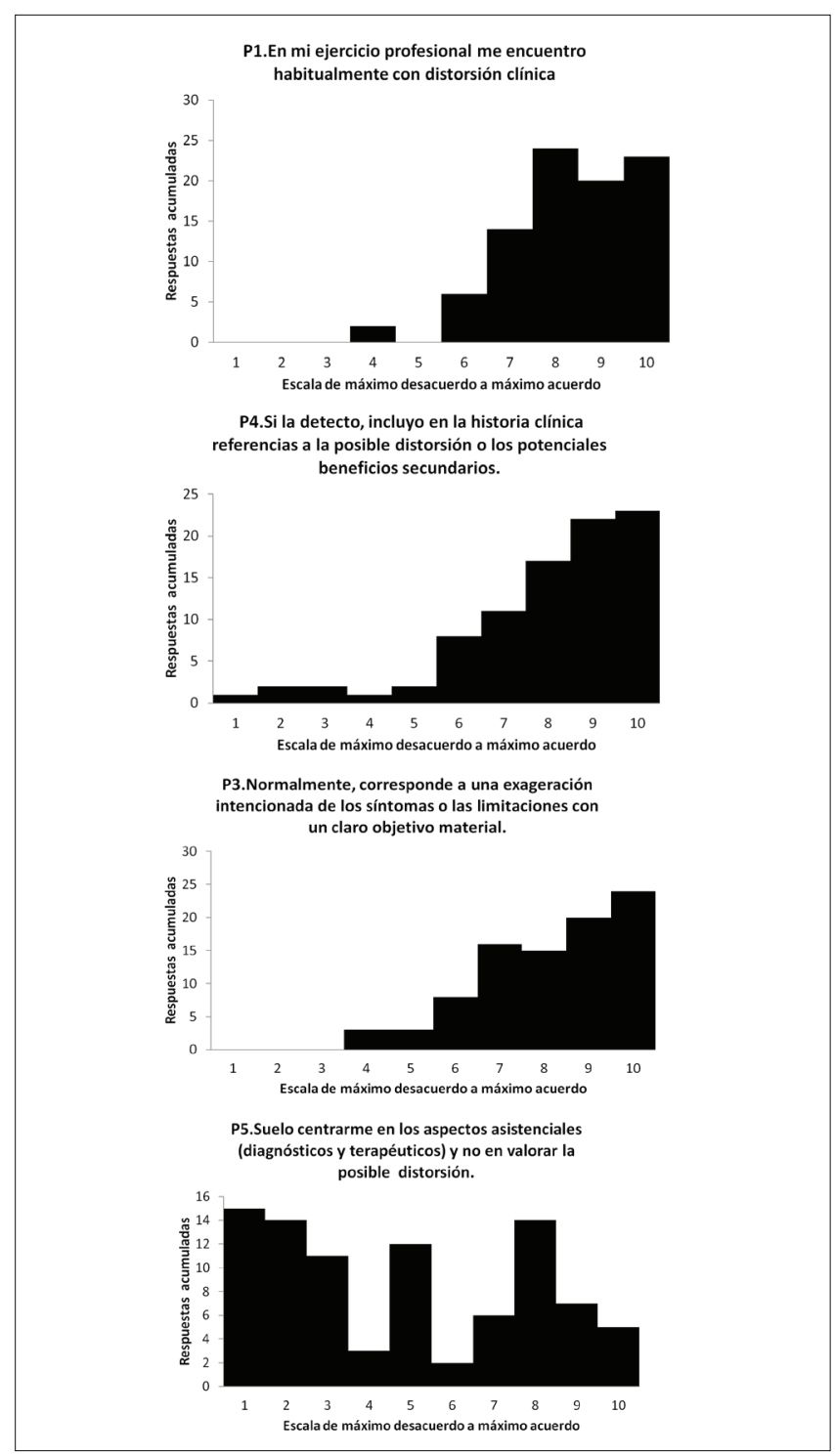

Figura 4: Distribución de respuestas a las Proposiciones acerca de la presencia de habitual de distorsión clínica (P1), su atribución a fraude por simulación (P2), la recogida efectiva de dichas circunstancias en la historia clínica (P4) o su ausencia (P5).

Entre las respondidas con No sabe/No contesta, destaca con gran diferencia la Proposición 13, relativa a los signos de Wadell en la exploración física, con 40 respuestas en la puntuación 5. Incluida en Controversias, existe una amplia producción bibliográfica a lo largo de las tres últimas décadas, que coincide en que dichos signos -inicialmente propuestos para detectar malos candidatos a cirugía por lumbociática y discopatías lumbares- no pueden considerarse indicativos de fraude por simulación, pero sí estarían claramente incluidos dentro del concepto de distorsión clínica que hemos explicado y hay estudios recientes que así lo muestran (Wygant et al., 2016). Es una controversia que el abordaje que planteamos resuelve; en presencia de signos de Waddell, debemos aplicar el diagnóstico diferencial de la distorsión clínica.

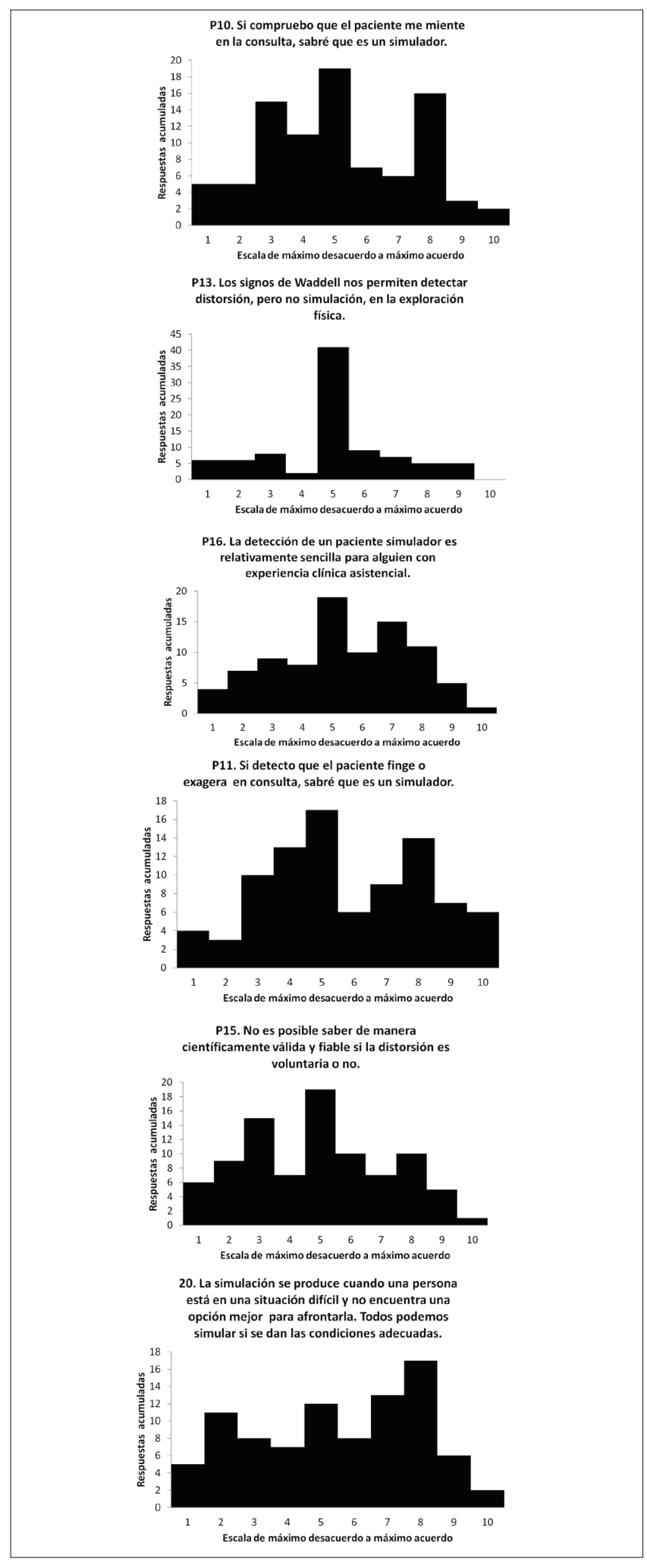

Figura 5: Distribución de respuestas a las Proposiciones acerca de la convicción de simulación en presencia de engaño (P10) o de exageración o fingimiento (P11), el significado de los signos de Wadell (P13), la incertidumbre (P15) en la atribución de voluntariedad en la distorsión y la dificultad de detectar el fraude por simulación (P16) y acerca del modelo adaptativo para explicarlo. 
Por último, destacaremos la Proposición 5- realización de comprobaciones acerca de la consistencia del caso- de entre las que tienen respuestas muy distribuidas, como se comprueba en su gráfico. Nos llama especialmente la atención ya que, en principio, cualquier perito médico -sea o no inspector- debería considerar dichas comprobaciones como una parte ineludible de su actuación médico-pericial. De ahí que esta Proposición forme parte de la categoría Práctica Diaria y no, por ejemplo, Controversias. Este resulta un aspecto clave porque obviar el análisis de validez puede suponer, desde nuestro punto de vista, lesionar algunos de los Principios bioéticos. No estamos hablando, como pudiera pensarse, exclusivamente del principio de Justicia, habitualmente invocado en estos casos. La renuncia a detectar y gestionar la distorsión y el potencial fraude en valoración de incapacidad supone permitir la percepción de prestaciones económicas por quienes no las precisan, lo que-mediante el condicionamiento operante- contribuirá a consolidar situaciones de limitación e invalidez que previamente no eran tales, al reforzar una conducta de padecimiento o rol de enfermo asumido de manera más o menos voluntaria. Tanto la sociedad en conjunto como el trabajador saldrían perdiendo en estas situaciones, que lesionan los principios de Beneficencia y No Maleficencia, como un parasitismo que envenena al que lo realiza, que hemos Ilamado Síndrome de Invalidez Aprendida (Domínguez et al., 2014).

A pesar de todo ello, la complejidad que puede suponer distinguir entre las diversas situaciones de distorsión clínica descritas y sus repercusiones personales, éticas y sociales explican, entre otros motivos, que actualmente el abordaje del problema se realice -o no- de manera individual según cada profesional implicado y, en general, de forma intuitiva y no estructurada.

Revisión de la bibliografía de referencia en medicina evaluadora: En las versiones revisadas del Manual de Actuación para Médicos del INSS $(2003 ; 2011)$ aparece un apartado de unas veinte líneas sobre la simulación en el tema dedicado a la Psiquiatría, una frase en el de Oncología ("En la valoración del paciente oncológico no suele estar presente la simulación") y menciones en el estudio neuropsicológico, las medidas objetivas de audición, los problemas de la ergometría convencional en Cardiología y las indicaciones de las pruebas biomecánicas en Ap. Locomotor. En la Guía de Valoración de Incapacidad Laboral para Médicos de Atención Primaria (Álvarez et al., 2009), las referencias son las mismas, excepto las menciones. En los Cursos de Actualización en Medicina de Evaluación, revisados, solo en 2012-2013 hubo un tema, especialmente interesante, Ilamado "Simulación en Psiquiatría" que estaba muy orientado a la aplicación de los resultados del MMPI-2 (Minnesota Multiphasic Personality Inventory-2) en aquellos casos en los que se sospecha fraude por simulación. Desgraciadamente, no en todas las Unidades Médicas se dispone de la posibilidad de solicitar el estudio neuropsicológico que incluya el MMPI-2, aunque este sería uno de los aspectos susceptibles de mejora, integrado en el análisis de validez.

Comunicamos este estudio en el II Congreso Nacional de Médicos Inspectores de la Administración de la Seguridad Social, celebrado en 2013 en Madrid y después hemos continuado con esta línea de trabajo. Para ello, partiendo de la habitual bibliografía médica de referencia nacional (González et al., 2012) e internacional (Rogers, 2008) estamos tratando de aplicar un enfoque multidisciplinar (Domínguez et al., 2015) incorporando técnicas de la Criminología o la Psicología Forense, como el Análisis de Conducta, incluido el perfilado de personalidad y el comportamiento no verbal.

\section{Conclusiones:}

1. Los inspectores médicos evaluadores del INSS, quizá por su distinta formación profesional, experiencia previa y práctica diaria en las diferentes Unidades Médicas, aparecen como un colectivo diverso.

2. Consideran que la distorsión clínica es una situación frecuente relevante y en sus consultas. El subtipo más frecuente correspondería al fraude por simulación.

3. Pese a que se trata de uno de los elementos nucleares de su actividad, los inspectores médicos no parecen contar actualmente con referencias específicas suficientes ni con un discurso científico común estructurado para estos casos, que son abordados según criterios individuales más o menos ajustados al estado actual del conocimiento.

4. La necesaria formación específica precisaría desarrollar un marco teórico que se beneficiará de integrar el conocimiento científico y práctico obtenido desde otras disciplinas que abordan la distorsión, el engaño y el fraude como la Psicología Social, Conductual y Forense, la Criminología y Ciencias de la Seguridad y las propias Ciencias Jurídicas. Por ello, planteamos el Análisis de Conducta como marco integrador.

\section{Referencias}

Álvarez-Blázquez Fernández F, Jardón Dato E, Carbajo Sotillo MD, Terradillos García MJ, Valero Muñoz MR, Robledo Muga F, Maqueda Blasco J, Cortés Barragán R \& Veiga de Cabo J. (2009). Guía de Valoración de Incapacidad Laboral para Médicos de Atención Primaria. Escuela Nacional de Medicina del Trabajo (ENMT)-Instituto de Salud Carlos III, Madrid. Consultada en http://www.isciii.es/ISCIII/es/ contenidos/fd-publicaciones-isciii/fd-documentos/GUIA_DE_VALORACION_DE_INCAPACIDAD_LABORAL_PARA_AP.pdf 
Álvarez Sáenz JJ, Álvarez Collado L \& Álvarez Collado CJ. (2003). La pericial médica. El médico evaluador del Instituto Nacional de la Seguridad social como perito titular. Medicina y Seguridad del Trabajo 193, 59-62.

Álvarez, F.-Blázquez Fernández, D., de coordinación Emilio Jardon Dato, E., Dolores Carbajo Sotillo, M., Jesús Terradillos García, M., Rosario Valero Muñoz, M., Robledo Muga, F. \& Herrero, V. L. (2012). Guía de valoración de incapacidad laboral para médicos de atención primaria. Madrid. Consultada en http://www.isciii.es/ISCIII/es/ contenidos/fd-publicaciones-isciii/fd-documentos/GUIA_DE_VALORACION_DE_INCAPACIDAD_LABORAL_PARA_AP.pdf

American Psychiatric Association. Task Force on DSM-IV. (2000). Diagnostic and statistical manual of mental disorders : DSM-IV-TR. Washington DC: American Psychiatric Association. Consultada en http://www.worldcat.org/title/diagnostic-and-statistical-manual-of-mental-disorders-dsm-iv-tr/oclc/43483668

Aronoff, G. M. (1991). Chronic pain and the disability epidemic. The Clinical Journal of Pain, 7(4), 330-8. Consultada en http://www.ncbi.nlm.nih.gov/pubmed/1809447

Arancón Viguera A. (2004). Teoría y práctica de la medicina evaluadora. Mapfre, Madrid.

Briñol Turnes P, de la Corte Ibáñez L \& Becerra Grande A. (2001). ¿Qué es persuasión? Biblioteca Nueva, Madrid.

Burgos-Ruiz de Castroviejo S, Díaz-García A M \& Roldán-León J. (2008). Valoración de la Incapacidad Laboral. Equipo de Valoración de Incapacidades. Rehabilitación 50, 291-314.

Domínguez Muñoz A, López Pérez R, Gordillo León F, Perez-Nieto MA, Gómez Álvarez A \& de la Fuente Madero JL. (2013). Distorsión clínica y simulación en la incapacidad temporal; un estudio preliminar. Psicopatología Clínica, Legal y Forense 13, 29-45. http://masterforense.com/pdf/2013/2013art2.pdf

Domínguez Muñoz A. (2014). La simulación como fraude a la Seguridad Social: Reflexiones sobre estudios previos. Comunicación oral al X Congreso Español de Criminología, Granada.

Domínguez Muñoz, A., López Pérez, R., Gordillo León, F., Perez-Nieto, MA., Gómez Álvarez A. \& de la Fuente Madero, J.L. (2014). Bases científicas y bioéticas del Análisis de Validez en Medicina Evaluadora. Medicina y Seguridad del Trabajo 236, 527-535. http://scielo.isciii.es/pdf/mesetra/v60n236/inspeccion2.pdf
Domínguez Muñoz A. Gómez Álvarez A. de la Fuente Madero JL. López Pérez R. Gordillo León F. \& Pérez-Nieto MA. (2015) Deshonestidad, engaño clínico y fraude; un área de mejora potencial en medicina pericial desde la interdisciplinariedad. Comunicación al III Congreso de Médicos Inspectores de la Administración de la Seguridad Social, Madrid.

Domínguez-Muñoz A. (2016). Utilidad del análisis del CNV en Medicina Pericial. En Comportamiento no verbal: más allá de la comunicación y el lenguaje, ed. López RM, Gordillo F \& Grau M, pp. 239-244. Pirámide.

González-Ordi H, Santamaría P \& Capilla-Ramírez P. (2012). Estrategias de detección de la simulación. Un manual clínico multidisciplinar. 1a Ed.TEA Ediciones, Madrid.

INSS (2003) Manual de actuación para médicos del INSS. Secretaría Estado de la Seguridad Social-Ministerio de Trabajo y Asuntos Sociales, Madrid.

INSS (2011) Manual de actuación para médicos del INSS. Secretaría Estado de la Seguridad Social-Ministerio de Trabajo e Inmigración, Madrid.

Ley 41/2002, de 14 de noviembre, básica reguladora de la autonomía del paciente y de derechos y obligaciones en materia de información y documentación clínica. Boletín Oficial del Estado núm. 274 de 15 de noviembre de 2002. Gobierno de España. https://www.boe.es/diario_boe/txt.php?id=BOE-A-2002-22188

Rogers R. (2008). Clinical Assessment of Malingering and Deception.

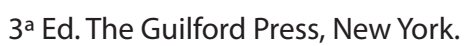

Vicente Herrero MT, Ramírez Iñiguez de la Torre MV, Capdevila García L, López González AA, Terradillos García MJ, Aguilar Jiménez E \& Torres Alberich Jl (2012). El concepto de daño laboral en España y su comparativo internacional: revisión legislativa Española, Hispano-Americana y Europea. Revista CES Salud Pública 3, 73-93.

Wygant DB, Arbisi PA, Bianchini, KJ, \& Umlauf RL. (2017). Waddell non-organic signs: new evidence suggests somatic amplification among outpatient chronic pain patients. Spine J 17, 505-510. 